\title{
BINARY STATISTICS AMONG POPULATION II STARS
}

\author{
H. Zinnecker, ${ }^{1}$ R. Köhler, ${ }^{2}$ and H. Jahreiß ${ }^{3}$ \\ RESUMEN
}

Las estrellas de Población II son estrellas viejas, pobres en metales, pertenecen al halo galáctico y tienen movimientos propios grandes. Hemos realizado una búsqueda de binarias visuales entre 164 estrellas del halo en la vecindad solar (distancia media $100 \mathrm{pc}$ ), mediante interferometría de motas en el infrarrojo, óptica adaptativa e imágenes directas de gran campo. La muestra se basa en las listas de Carney et al. (1994) y Norris (1986), con distancias confiables del HIPPARCOS. En primera instancia, hemos encontrado 33 binarias, 6 triples y una cuádruple. Si nos limitamos a cocientes de flujo en la banda K mayores que 0.1 (para evitar contaminación por el fondo) los números bajan a 9 binarias y una triple, lo cual corresponde a una fracción de binarias del 6 al $7 \%$, por arriba de nuestro límite de resolución angular de 0.1 segundos de arco. Si contamos todas las binarias con cocientes de flujo en la banda $\mathrm{K}$ mayores que 0.01 , obtenemos 15 binarias y 3 triples adicionales, lo cual corresponde a una fracción de binarias de alrededor del $20 \%$ para separaciones proyectadas mayores que unas $10 \mathrm{AU}$. Para efectos de comparación, la frecuencia de binarias espectroscópicas para Población II es de $15 \%$ (para períodos de hasta 3000 días) (Latham et al. 2002). También hemos determinado la distribución de semiejes mayores para nuestras binarias y triples visuales de Población II. Observaciones en una segunda época servirán para confirmar la realidad de nuestros resultados.

\section{ABSTRACT}

Population II stars are old, metal-poor, Galactic halo stars with high proper motion. We have carried out a visual binary survey of 164 halo stars in the solar neighborhood (median distance $100 \mathrm{pc}$ ), using infrared speckle interferometry, adaptive optics, and wide field direct imaging. The sample is based on the lists of Population II stars of Carney et al. (1994) and Norris (1986), with reliable distances from HIPPARCOS measurements.

At face value, we found 33 binaries, 6 triples, and 1 quadruple system. When we limit ourselves to K-band flux ratios larger than 0.1 (to avoid background contamination), the numbers drop to 9 binaries and 1 triple, corresponding to a binary frequency of $6-7 \%$ above our angular resolution limit of about 0.1 arcsec. If we count all systems with K-band flux ratios greater than 0.01 , we obtain 15 more binaries and 3 more triples, corresponding to a binary frequency for projected separations in excess of $10 \mathrm{AU}$ of around $20 \%$. This is to be compared with the frequency of spectroscopic binaries (up to a period of 3000 days) of Population II stars of about 15\% (Latham et al. 2002). We also determined a semi-major axis distribution for our visual Population II binary and triple systems, which appears to be remarkably different from that of Population I stars. Second epoch-observations must help confirm the reality of our results.

Key Words: TECHNIQUES: HIGH ANGULAR RESOLUTION - BINARIES: VISUAL — INFRARED: STARS

\section{INTRODUCTION}

Along with the Initial Mass Function, the frequency of binary stars and the distribution of their periods and mass ratios are among the key observable statistics of the star formation process. The main reason or assumption is that the initial binary parameters are practically frozen after the formation phase, thus providing us with a fossil record of binary star formation.

Studies of binaries among nearby solar-type

\footnotetext{
${ }^{1}$ Astrophysikalisches Institut Potsdam, Germany.

${ }^{2}$ MPI für Astronomie, Heidelberg, Germany.

${ }^{3}$ Astronomisches Recheninstitut, Heidelberg, Germany.
}

main-sequence stars show that about $53 \%$ of them are binary or multiple systems (Duquennoy \& Mayor 1991), while the fraction is $42 \%$ among nearby Mstars (Fischer \& Marcy 1992). Surveys of lowmass pre-main-sequence stars in the star-forming region Taurus-Auriga found a binary frequency of $80-$ $100 \%$, depending on how the extrapolation to unresolved systems is made (Leinert et al. 1993, Ghez, Neugebauer \& Matthews 1993, Köhler \& Leinert 1998). Binary studies of southern star-forming regions, e.g. Scorpius-Centaurus, give similar results (e.g. Simon 1995, Brandner \& Köhler 1998, Köhler et al. 2000). These results indicate that binaries are 
the rule and not the exception in the outcome of star formation (Mathieu 1994).

All these studies dealt with Population I stars that formed within the Galactic disk. Population II stars are older and formed in the Galactic halo, i.e. in a different environment. They are characterized and identified by their high proper motions and low metallicities, reflecting the more turbulent and less metal-enriched gaseous raw material from which stars formed in the very early phases of our Galaxy. We can expect that the different initial conditions of Population II star formation has led to differences in the binary frequency or the distributions of separations and mass ratios among Population II stars compared to Population I stars. This hypothesis can now be tested for the first time, since HIPPARCOS has given us good parallaxes for the Population II stars, necessary to convert any measured angular separation of binary stars into physically meaningful linear separations and also to convert apparent magnitudes of Population II stars into luminosities and hence masses (for the mass-luminosity relation of Population II main-sequence stars, see Kroupa \& Tout 1997 and Baraffe et al. 1997).

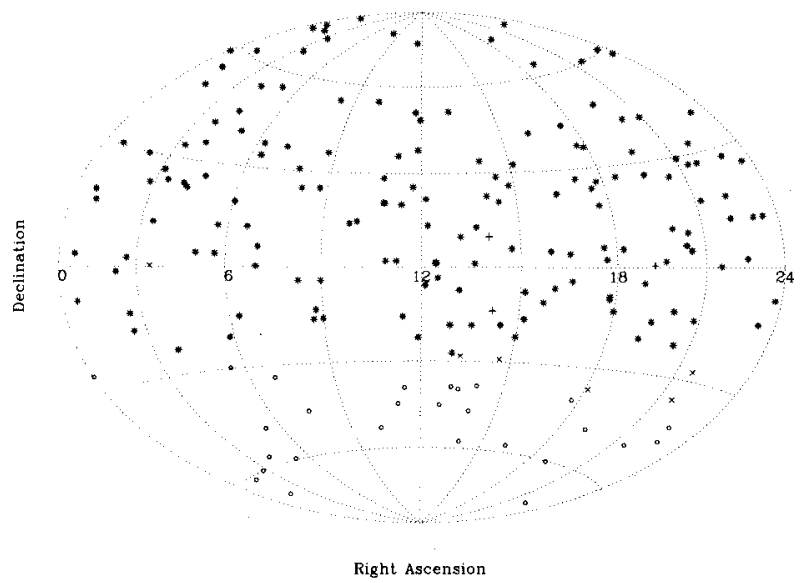

Fig. 1. Distribution of the stars in our sample on the sky. Stars marked by + have been observed with speckle or Adaptive Optics techniques, stars marked by $x$ with standard seeing-limited imaging, and stars marked by 0 have not been observed by us so far.

\section{SAMPLE SELECTION AND OBSERVATIONS}

In the years 1997-2001, we carried out a high spatial resolution multiplicity survey of about 200 Population II stars, using speckle interferometry and direct imaging on Calar Alto, and the Adaptive Optics system at Lick Observatory, California. All the observations were taken in the near-infrared K-band at 2.2 micron. The sample was selected from the lists of Population II stars of Carney et al. (1994)

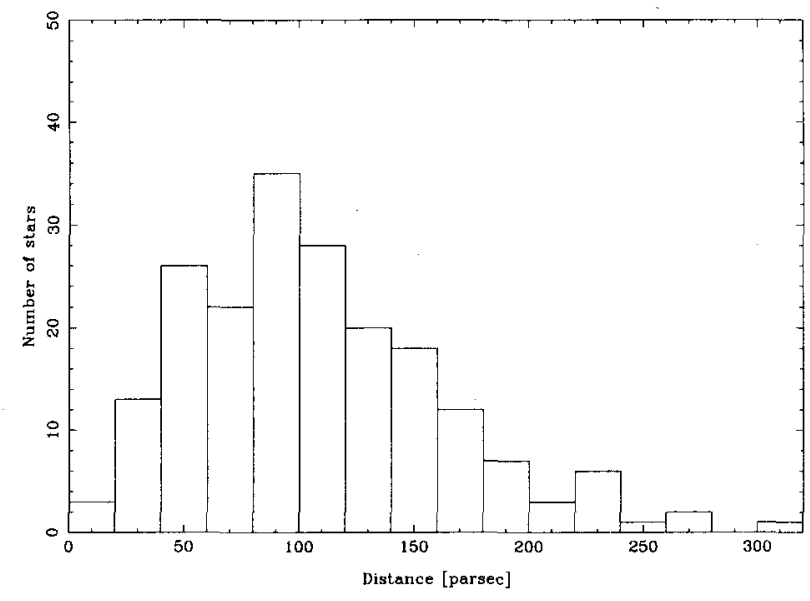

Fig. 2. Distance distribution of the stars in our sample, as measured by HIPPARCOS.

in the northern hemisphere and Norris (1986) in the southern hemisphere, i.e. the subset with both high space motions $(>200 \mathrm{~km} / \mathrm{s})$ and low metallicities $([\mathrm{Fe} / \mathrm{H}]<-1.6)$. Of those "extreme halo stars" we kept only the ones for which cross-identification with the HIPPARCOS catalogue (ESA 1997) provided an accurate parallax, resulting in a total of 197 Population II targets. Of these, 164 have been observed (the missing 33 objects are all located deep in the southern hemisphere, i.e. below $\delta=-30^{\circ}$ ). The distribution of our sample on the sky is shown in Fig. 1, their distribution in distance in Fig. 2. The data were reduced using the speckle data reduction package discussed in Köhler et al. (2000). The kinematics of halo subdwarfs from Carney et al. (1994) with reliable HIPPARCOS parallaxes and proper motions is discussed in Jahreiß, Fuchs \& Wielen (1997) and Fuchs, Jahreiß, \& Wielen (1999) who distinguish three sub-populations, one of which is "our" non-rotating extreme halo population.

\section{RESULTS}

At face value, we find 48 companions among 164 stars observed, 33 binaries, 6 triples, and possibly 1 quadruple system. When we limit ourselves to Kband brightness ratios larger than 0.1 (to avoid background contamination), the numbers drop to 9 binaries (HIP 17666, HIP 18235, HIP 59233, HIP 66665, HIP 71979, HIP 80003, HIP 88010, HIP 111549, HIP 114962) and 1 triple system (HIP 28671), corresponding to a companion star frequency of $6-7 \%$ above our angular resolution limit of about 0.1 arcsec (10 AU). If we relax our K-band flux ratio limit to greater than 0.01 , we obtain 15 more binaries and 3 extra triple systems (HIP 92781, HIP 94449, HIP 105488), for a total of 32 companions, corresponding 
to a possible binary frequency of around $20 \%$ down to the same resolution limit. The projected separations of these 32 companions are plotted in Fig. 3 and exhibit a bimodal distribution. The bimodality also remains after a statistical correction for chance alignment with background stars has been applied. This was done using field star counts in the direct, seeing-limited images from the $1.23 \mathrm{~m}$ and $2.2 \mathrm{~m}$ Calar Alto observations. This procedure is subject to considerable uncertainty, as the field star density had to be determined for each of the widely spread targets separately.

\section{DISCUSSION AND OUTLOOK}

It is important to check whether the peak at separations of about $500 \mathrm{AU}$ is real. If it is, the separation distribution would be bimodal, perhaps indicating two different (hard and soft) sub-populations (cf. Weinberg, Shapiro, \& Wasserman 1987) However, it is too early to speculate about stellar dynamical processes that could lead to this bimodality, as long as its reality is not firmly and safely established.

By far the best way to clarify this issue is a second-epoch measurement of the Population II binary systems in the relevant separation range. We are going to obtain such observations for some 40 wide Population II stars using the MAGIC NIRcamera at the Calar Alto $2.2 \mathrm{~m}$ telescope, at $0.66^{\prime \prime}$ per pixel, to see if the positions of the companions have changed relative to the primaries or not. If the relative positions did not change by much, the two stars form a common proper motion pair and they are a bound system.

The proper motions of our objects are well known from HIPPARCOS, and all objects are high propermotion stars. Indeed the time interval since the first-epoch discovery observations is large enough ( $~ 5$ years) to measure the change $(>0.5 ")$ of the relative positions of spurious, optical companions with MAGIC. The periods of true common proper motion binaries are also long enough (hundreds of years) so that orbital motion will not interfere.

Measuring the frequency of common proper motion pairs for our Population II sample and comparing it with Population I field stars in the solar neighborhood will provide further insight into the primordial or evolutionary processes that operate at the wide end of the semi-major axis distribution of field Population II binaries (cf. Allen, Poveda, \& Herrera 2000). Perhaps it can help answer another fundamental question: did all the extreme Populations II field stars originally form in dense, but now long dissolved globular clusters? If so, no or only few Pop-



Fig. 3. Distribution of separations of all companions found in our Population II multiplicity survey. The linear separations of Population II stars (Histograms) were computed from the angular separations measured by us and the distance to the individual objects measured by HIPPARCOS. The open histogram shows the uncorrected numbers of companions found, the hatched histogram is the result of correction for chance alignment with background stars. The distribution of Population I stars (gaussian-shaped curve) is the result of Duquennoy \& Mayor (1991) for G-dwarfs in the solar neighborhood. The distribution of Population I M-dwarfs (Fischer \& Marcy 1992) is virtually indistinguishable from it.

ulation II common proper motion pairs should be found! What do you think, Professor Poveda?

\section{ACKNOWLEDGEMENTS}

We would like to congratulate Arcadio Poveda for his scientific achievements and the inspiration he provided for Mexican astronomy. It was a great idea to celebrate his achievements in Merida, his home city. We, the binary star community, owe special thanks to the co-chairs of the SOC, Christine Allen and Colin Scarfe, for their initiative to seize the occasion to bring us all together in what turned out to be a memorable IAU Colloquium. Many thanks also to the LOC for taking us on those splendid Mayan excursions to the nearby archaeo-astronomical sites of Uxmal and Chichen-Itza. This work has been supported in part by the German Science Foundation (DFG) and the National Science Foundation Science and Technology Center for Adaptive Optics, managed by the University of California at Santa Cruz under cooperative agreement No. AST-9876783. 
Note added in proof: This paper is a continuation of our first preliminary reports on the subject given in Köhler, Zinnecker, \& Jahreiß $(2000,2001)$. The binary frequency of high-velocity halo field subdwarfs has been studied by a number of authors over the last two decades, including the early work by Stryker et al. (1985) and Abt \& Willmarth (1987). More recently, Gizis \& Reid (2000) and particularly Latham et al. (2002) made significant contributions, i.e. a small HST snapshot imaging survey and an extensive spectroscopic investigation, respectively. While the early work claimed some deficit of Population II binaries with respect to Population I, the most recent publications seem to put these claims to rest.

\section{REFERENCES}

Abt, H. A. \& Willmarth, D. W. 1987, ApJ, 318, 786

Allen, C., Poveda, A., \& Herrera, M. A. 2000, A\&A, 356, 529

Baraffe, I., Chabrier, G., Allard, F., \& Hauschildt, P. H. 1997, A\&A, 327, 1054

Brandner, W. \& Köhler, R. 1998, ApJ, 499, L79

Carney, B. W., Latham, D. W., Laird, J. B., \& Aguilar, L. A. 1994, AJ, 107, 2240

Duquennoy, A., \& Mayor, M. 1991, A\&A, 248, 485

Fischer, D. A., \& Marcy, G. W. 1992, ApJ, 396, 178

Fuchs, B., Jahreiß, H., \& Wielen, R. 1999, Ap\&SS, 265, 175
Ghez, A. M., Neugebauer, G., \& Matthews, K. 1993, AJ, 106,2005

Gizis, J. E., \& Reid, I. N. 2000, PASP, 112, 610

Jahreiß, H., Fuchs, B., \& Wielen, R. 1997, in Proc. HIPPARCOS Venice' 97 , ed. M. A. C. Perryman \& P. L. Bernacca, ESA SP-402, 587

Köhler, R., \& Leinert, Ch. 1998, A\&A, 331977

Köhler, R., Kunkel, M., Leinert, Ch., \& Zinnecker, H. 2000, A\&A, 356, 541

Kroupa, P., \& Tout, C. A. 1997, MNRAS, 287, 402

Köhler, R., Zinnecker, H., \& Jahreiß, H. 2000, in IAU Symp. 200 (posters), Birth and Evolution of Binary Stars, ed. B. Reipurth \& H. Zinnecker, 148

Köhler, R., Zinnecker, H., \& Jahreiß, H. 2001, in Dynamics of Star Clusters and the Milky Way, ed. S. Deiters, B. Fuchs, A. Just, R. Spurzem, \& R. Wielen, ASP Conf. Ser., 228, 491

Latham, D. W., Stefanik R. P., Torres, G., Davis, R. J., Mazeh, T., Carney, B. W., Laird, J. B., \& Morse, J. A. 2002, AJ, 124, 1144

Leinert, Ch., Zinnecker, H., Weitzel, N., Christou, J., Ridgeway, S. T., Jameson, R., Haas, M., \& Lenzen R. 1993, A\&A, 278, 129

Mathieu, R.D. 1994, ARA\&A, 32, 465

Norris, J. 1986, ApJS, 61, 667

Simon M. 1995, Ap\&SS, 223, 45

Stryker, L. L., Hesser, J. E., Hill, G., Garlick, G. S., \& O'Keefe, L. M. 1985, PASP, 97, 247

Weinberg, M. D., Shapiro, S. L. \& Wasserman, I. 1987, ApJ, 312, 367

Hans Zinnecker: Astrophysikalisches Institut Potsdam, An der Sternwarte 16, 14482 Potsdam, Germany (hzinnecker@aip.de).

Rainer Köhler: Max-Planck-Institut für Astronomie, Königstuhl 17, 69117 Heidelberg, Germany (koehler@ mpia.de).

Hartmut Jahreiß: Astronomisches Recheninstitut, Mönchshofstr. 12-14, 69120 Heidelberg, Germany (jahreiss@ ari.uni-heidelberg.de). 